Regular Articles

\title{
A practical approach for optical characterization of a film coated on the optical fiber 出, 败识
}

\author{
Çağın Ekici*, Mehmet Salih Dinleyici \\ Department of Electrical and Electronics Engineering, Izmir Institute of Technology, Izmir, Turkey
}

\section{A R T I C L E I N F O}

\section{Article history:}

Received 1 November 2016

Revised 28 April 2017

Accepted 14 May 2017

Available online 6 June 2017

\section{Keywords:}

Optical phase diffraction

Dielectric polymer film optical properties

Fiber sensor

\begin{abstract}
A B S T R A C T
Phase Diffraction (PD) ${ }^{1}$ which is a result of the interaction of light waves with a transparent object, is exploited to characterize precisely optical properties of dielectric films coated on the optical fiber without harming any feature of the sample. Typical fiber sensor applications require films coated on the side surface of the optical fiber and optical properties of that curved films are crucial for design purposes. In this study, three Polyvinyl Alcohol (PVA) films are prepared, their thicknesses are estimated based on the phase diffraction method by fitting experimental results with a mathematical model within $2.3 \%$ error. The outcomes of this practical method show good agreement with findings of the destructive Scanning Electron Microscopy (SEM) measurements. The method has the potential to allow real time monitoring abrupt changes of surrounding medium's properties and to examine coating quality (i.e. thickness uniformity) of the film.
\end{abstract}

(c) 2017 Elsevier Inc. All rights reserved.

\section{Introduction}

Optical films are widely used for modulating light in various scientific and practical applications, such as optoelectronics, integrated optics, semiconductor technologies, and especially for optical sensors. Thus, precise measurement of the film properties (i.e. thickness and refractive index) is essential to obtain better optical performance. It becomes challenging process in recent years due to nanometer scale of film thickness requirements which is comparable or shorter than wavelengths of visible light. Conventional optical methods to characterize optical properties of films are based on interferometric methods [1,2] and ellipsometric methods $[3,4]$. For the curved substrates as in [3], change of the polarization state is interrogated, it is a complicated procedure. Furthermore, laboratory environment requirement of aforementioned methods results in preventing effective engineering fabrication.

Diffraction of a plane wave from a phase object is very rich subject and brings wealth of application, since phase of the wave is more sensitive to refractive index change than the amplitude. Accordingly, the resultant phase diffraction pattern is contributed

\footnotetext{
This document is a collaborative effort.

the second title footnote which is a longer than the first one and with an intention to fill in up more than one line while formatting.

* Corresponding author.

E-mail addresses: caginekici@iyte.edu.tr (Ç. Ekici), salihdinleyici@iyte.edu.tr (M. S. Dinleyici).

1 Phase Diffraction.
}

by the phase variation of transversing wave front through the curved film. Since the curved geometry globally modifies the wave front, features of the diffraction pattern (e.g. main lobe width) is the reflection of the film properties (e.g. quality, thickness). The approach is adapted for optical fiber based narrow dielectric films in this study.

Typical optical fiber sensor applications including surface plasmon resonances require dielectric polymer films (PVA etc.) coated on side surface of an optical fiber [5-7]. Therefore, nondestructive characterization of these films is crucial for designing, implementing, and monitoring such devices.

This letter presents the experimental results and estimations from a mathematical model of the diffraction of plane waves from a dielectric film coated on the optical fiber. When a light wave traverses the dielectric film, the particular regions of the wave front passing through peculiar sections of the film undergo specific phase delays as in Fig. 1. The region of the wave fronts passing through the phase object traced geometrically using optical ray approach [8] and more comprehensive studies have recently been done as well [9]. In [9], the intensity distribution of the light diffracted from phase steps of one and two dimensions in reflection and transmission modes are analyzed. A mathematical method based on Fresnel-Kirchhoff diffraction theory under paraxial approximation is exploited in this study. The diffraction pattern that is recorded by CCD camera is compared to results of the mathematical model to estimate thickness of the dielectric film coated on optical fiber provided that remaining parameters are known. The method can also be expanded for simultaneous estimation of 

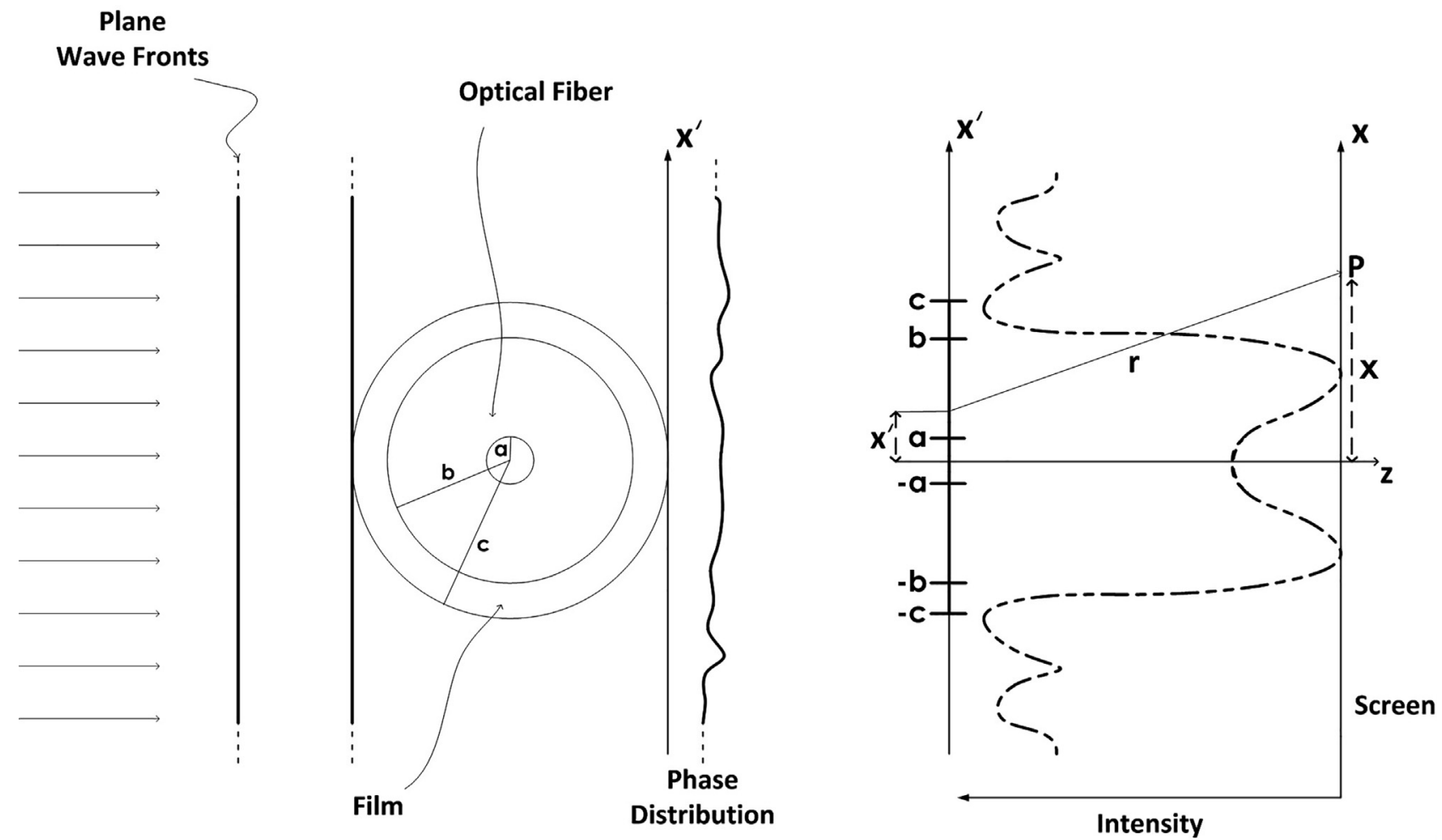

Fig. 1. Typical diffraction pattern of transmitted plane wave fronts through coated optical fiber and the diffraction geometry.

refractive index value of the film together with its thickness by using at least two different monochromatic light sources. Furthermore, this approach may be used for real time monitoring of changes in the surrounding medium properties (e.g. humidity level) which is the fundamental mechanism of many optical fiber sensor system.

Optical fibers are used as a cylindrical base in these works due to its perfect geometry, well defined optical properties, widespread usage, and easy accessibility. Apart from these, the method can easily be adapted for any kind of geometry that is transparent at the wavelength of interest. The approach was already applied for estimating the optical fiber core refractive index and its diameter [10] and adapted for a channel waveguide geometry which is laser direct-written in photopolymer substrates to characterize refractive index profile [11]. This letter propose a novel nondestructive practical approach for the curved dielectric films having their thicknesses comparable with the wavelength of interest and dimensions of the optical fiber.

\section{Mathematical model}

A mathematical model based on ray tracing and FresnelKirchhoff diffraction integral is adapted for the problem under paraxial approximation $[10,11]$. It is assumed that there is no optical variation along the optical fiber axis. Therefore, the problem can be considered only in the cross-section plane. After the optical rays propagating through different parts of the coated optical fiber, the uniform wave front points advance and exhibit a phase distribution. This process can be modeled by using ray tracing between the entering plane and the exit surface of the film. Then, the resultant wave front experiences diffraction (self interferences) between the exit surface of the film and an observation plane as shown in Fig. 1.

Optical fibers are considered as two layer geometry in the previous study [10]. Here, the model is extended for coated optical fibers (three layer geometry) by using the similar approach. The amplitude of the diffracted wave on the observation plane at an arbitrary point $P$ in Fig. 1:

$$
\begin{aligned}
U(P)= & K\{1+C(\gamma)-C(\xi)+j[1+S(\gamma)-S(\xi)] \\
& +\sqrt{\frac{2}{\lambda z}}\left(\int_{-c}^{-b} \exp \left(-j \phi_{p}\right) \exp \left[j k \frac{\left(x-x^{\prime}\right)^{2}}{2 z}\right] d x^{\prime}\right. \\
& +\int_{-b}^{-a} \exp \left(-j\left(\phi_{p}+\phi_{c l}\right)\right) \exp \left[j k \frac{\left(x-x^{\prime}\right)^{2}}{2 z}\right] d x^{\prime} \\
& +\int_{-a}^{a} \exp \left(-j\left(\phi_{p}+\phi_{c l}+\phi_{c o}\right)\right) \exp \left[j k \frac{\left(x-x^{\prime}\right)^{2}}{2 z}\right] d x^{\prime} \\
& +\int_{a}^{b} \exp \left(-j\left(\phi_{p}+\phi_{c l}\right)\right) \exp \left[j k \frac{\left(x-x^{\prime}\right)^{2}}{2 z}\right] d x^{\prime} \\
& \left.\left.+\int_{b}^{c} \exp \left(-j \phi_{p}\right) \exp \left[j k \frac{\left(x-x^{\prime}\right)^{2}}{2 z}\right] d x^{\prime}\right)\right\},
\end{aligned}
$$

in which $a, b$, and $c$ are the core, the cladding, and the coated optical fiber radii. $C$ and $S$ are Fresnel cosine and sine functions. Their dependency on $\gamma$ and $\xi$ indicate $\sqrt{2 /(\lambda z)}(x-c)$ and $\sqrt{2 /(\lambda z)}(x+c)$, respectively. Finally $K, \phi_{p}, \phi_{c l}$, and $\phi_{c o}$ are

$K=A \sqrt{\frac{-j}{2}} \exp (j k z) \exp \left(-j 2 k b n_{s}\right)$,

$\phi_{p}=2 k \sqrt{c^{2}-x^{\prime 2}}\left(n_{p}-n_{s}\right)$,

$\phi_{c l}=2 k \sqrt{b^{2}-x^{\prime 2}}\left(n_{c l}-n_{p}\right)$,

$\phi_{c o}=2 k \sqrt{a^{2}-x^{\prime 2}}\left(n_{c o}-n_{c l}\right)$,

where $A, n_{s}, n_{p}, n_{c l}, n_{c o}$, and $k$ are the amplitude of the disturbance, refractive indices of the surrounding medium, the coated material, the cladding, the core, and wave number, respectively. Light inten- 
sity is the main appraisement for almost all optical image formation systems and it can be calculated for the scalar fields as follows:

$I=U(P) U^{*}(P)$.

Dividing the above equation by the intensity at point $P$ in the absence of the coated optical fiber ends up with the normalized intensity. Diffraction pattern is obtained by depicting normalized intensity distribution versus $x$ which represents distance from the center of the diffraction pattern.

\section{Experimental methodology}

The optical fibers used in this work were coated with PVA to form three layer geometry. PVA is a water soluble polymer which is easy to turn into film due to excellent adhesion properties, was chosen in particular in this work for transparency at the wavelength of interest $(400-700 \mathrm{~nm})$ and fairly constant refractive index. The PVA was supplied by Sigma - Aldrich in granuler form (MW 30000-70000) and has a degree of hydrolysis of $87-90 \%$. PVA granules (dry) were mixed with water to form 15\% PVA solution. This PVA/water mixing was stirred approximately at $70{ }^{\circ} \mathrm{C}$ with the help of a magnetic stirrer for $10 \mathrm{~h}$ to allow PVA granules to completely dissolved. Thus, the PVA/water solution was prepared to coat fiber optics as thin film through evaporation of the solvent.

Dip coating method was used to produce PVA coated optical fiber due to its simplicity and effectiveness. Prior to the coating, optical fibers were cleaned using Isopropyl Alcohol and carefully dried. Optical fibers were first dipped into the solution and then drawn from the solution at constant speed and this process was repeated when a thicker coating was required. Afterwards, coated optical fibers were placed in an oven to be completely dried at $90{ }^{\circ} \mathrm{C}$ for two hours. The dried PVA film refractive index is expected to have $n_{p}=1.53$ [12].

A schematic of the experimental setup is sketched in Fig. 2. Experimental procedure began with conversion of the output of the $632.8 \mathrm{~nm}$ He-Ne laser light beam to a plane wave. Conventional beam expander was used for this purpose. Obtained plane waves were sent through the PVA coated optical fiber perpendicular to its axis. The diffraction patterns were recorded by CCD camera having $720 \times 640$ pixels image sensor with $11 \times 17 \mu \mathrm{m}^{2}$ pixel area. In addition to that, optical fiber specifications (factory data) are as follows: the fiber cladding and core radii are $2 \mathrm{~b}=125 \mu \mathrm{m}$, $2 \mathrm{a}=4.4 \mu \mathrm{m}$, respectively, refractive indices of the cladding and core are $n_{c l}=1.4537$ and $n_{c o}=1.4591$, respectively.

\section{Results and discussion}

Three samples were prepared using dip coating procedure as explained in the previous section. For three PVA films with varying thicknesses, the coating process was repeated once, twice, and three times for the first, second, and third sample, respectively. The diffraction patterns for these three samples were recorded by a CCD sensor located at $41 \mathrm{~mm}$ distance away. Verification of the procedure was completed by comparing the diffraction patterns with the estimations of the mathematical model. Conventional least-square method was used for the curve fitting purpose to estimate the value of the desired parameter (i.e. thickness).

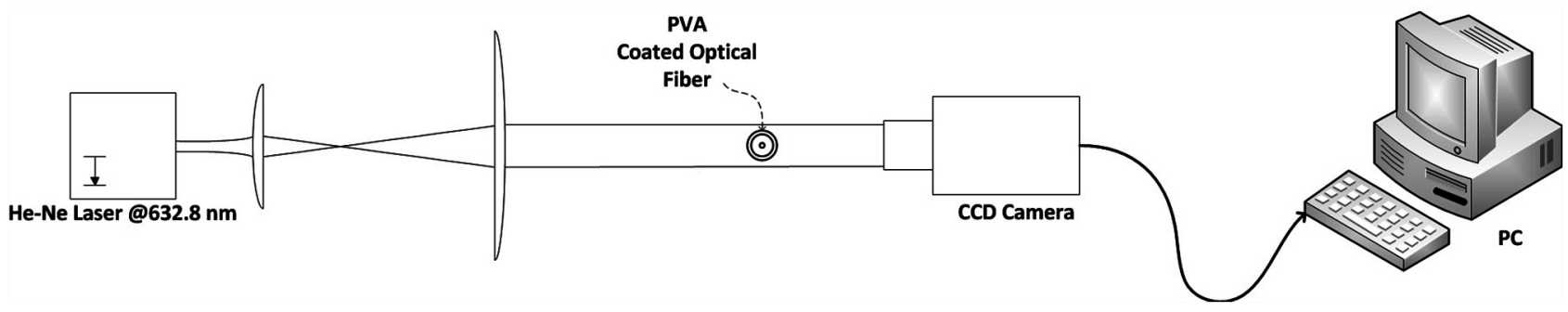

Fig. 2. Top view of experimental setup for estimating film thickness of a coated optical fiber.

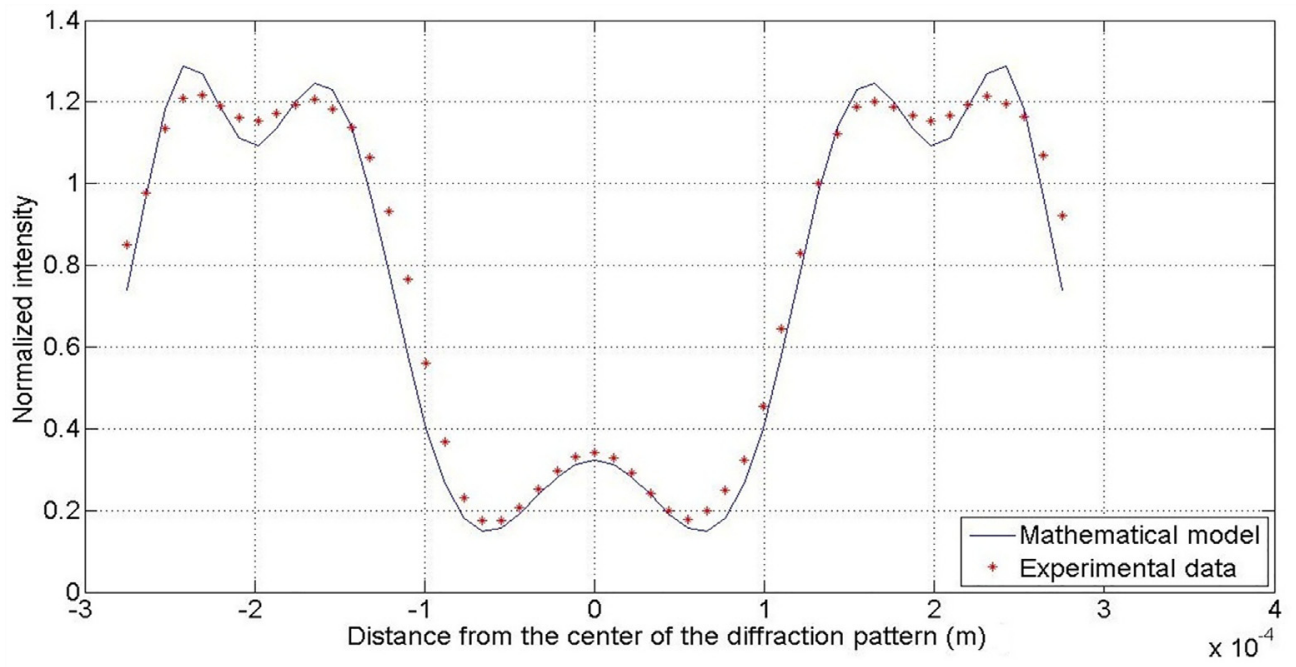

(a)

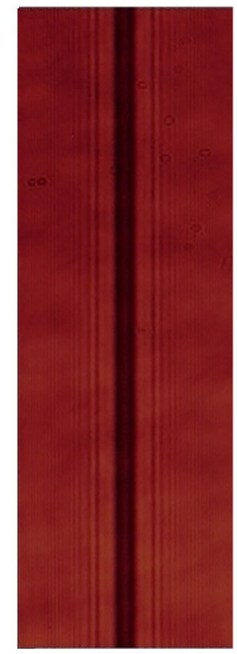

(b)

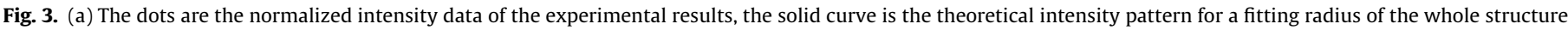
$63,429 \mathrm{~nm}$; (b) Diffraction pattern recorded by CCD camera of a plane wave diffracted by the PVA coated optical fiber. 


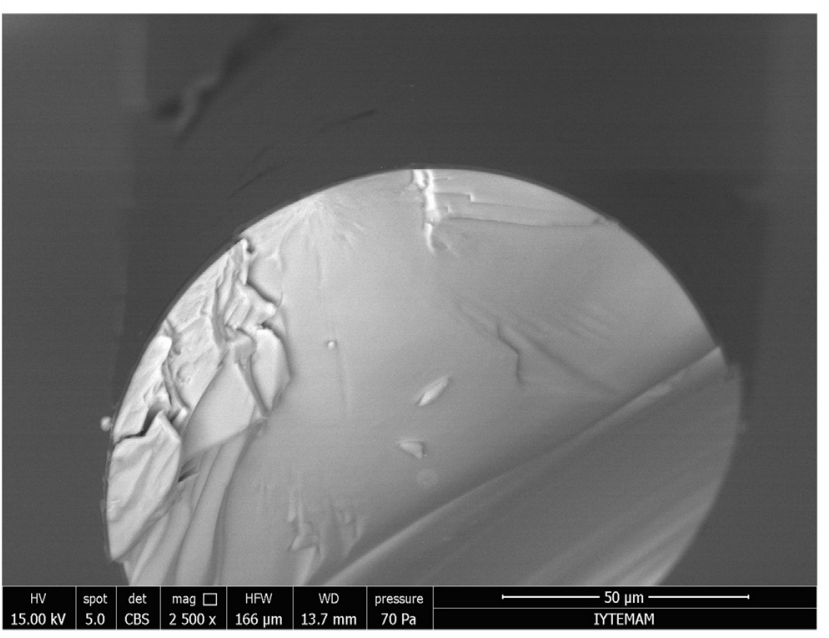

(a)

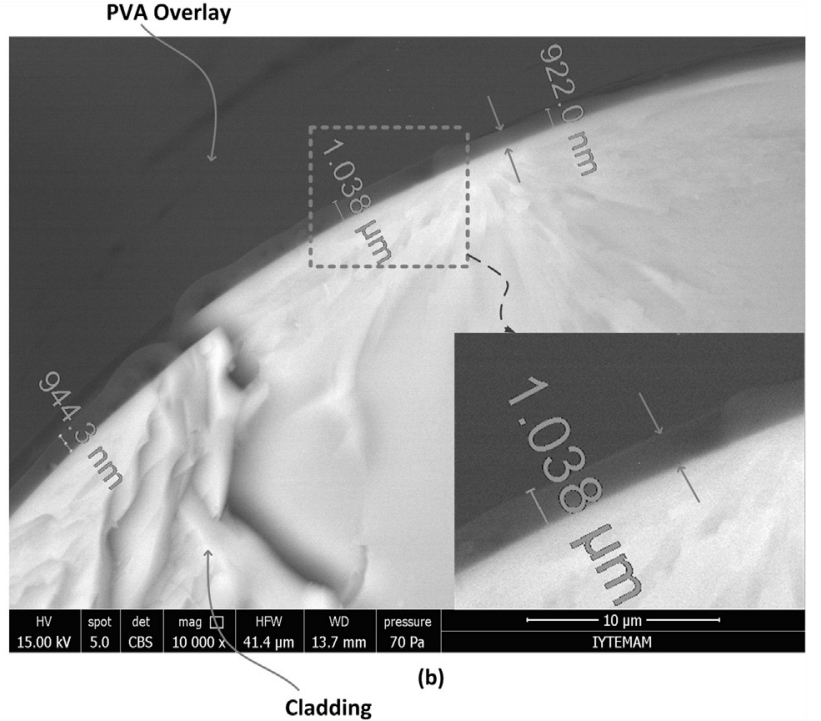

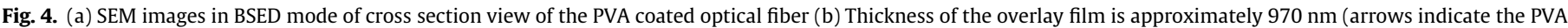
layer on the fiber).

The diffraction pattern measurement and the corresponding mathematical model conclusion of one of the PVA coated optical fiber is depicted in Fig. 3a) and its CCD image is shown in Fig. 3(b). The dots in the Fig. 3(a) are the normalized intensity data of the experimental results on the diffraction pattern and the solid curve is the model based intensity pattern for a fitting thickness parameter.

In order to prove and demonstrate the achievement of this approach the results are compared with the conclusion of destructive SEM technique, all three sample's SEM images were taken in BSED $^{2}$ mode. BSED simply responses dark or brighter in the sense of atomic number of area being analyzed. Thus, its thickness value may be read manually. Aforementioned optical fiber's SEM images are shown in Fig. 4. Fig. 4(a) is to show PVA film is coated uniformly; brighter area indicates cladding of the optical fiber, whereas dark area represents PVA film in Fig. 4(b).

The samples must be broken properly to examine manually in details by a SEM expert that is a tedious process. In contrast to SEM method, the nondestructive practical method is proposed here for the good of designing and implementing optical fiber based technologies.

Evaluated PVA film thicknesses for the three samples by using phase diffraction and their actual values according to SEM images are summarized in Table 1. Obviously error is inevitable and radius of errors are $13.3 \%, 4.2 \%$, and $2.3 \%$ for those whose actual PVA film thickness values are $508 \mathrm{~nm}, 970 \mathrm{~nm}$, and $1670 \mathrm{~nm}$, respectively.

\section{Conclusion}

This study demonstrates that dielectric transparent films coated on the curved surface can be practically characterized by analyzing the diffraction patterns. This nondestructive practical approach does not require any sophisticated equipments, and thus appropriate for designing a commercial device that performs characterization as well as real time monitoring. We experimentally show that the method can estimate as low as $2.3 \%$ error for a film $1670 \mathrm{~nm}$ thick.

Despite the fact that we evaluated $440 \mathrm{~nm}$ thick film on a typical telecom optical fiber using $632.8 \mathrm{~nm}$ light wave, in which boundary of the Abbe's diffraction limit $(\approx \lambda / 2)$, phase diffraction

\footnotetext{
2 Back-Scattered Electron Detector.
}

Table 1

Summary of coated optical fiber PVA film thickness values.

\begin{tabular}{lll}
\hline Number & Thickness $(\mathrm{nm})$ PD & Thickness $(\mathrm{nm})$ SEM \\
\hline Fiber \#1 & 440 & 508 \\
Fiber \#2 & 929 & 970 \\
Fiber \#3 & 1630 & 1670
\end{tabular}

method may allow to exceed that limit by observing reactive near fields (evanescent fields) [13]. Furthermore, the film quality can be deduced by recording and analyzing diffraction pattern in details with the help of signal processing techniques.

\section{Acknowledgements}

This work was supported in part by the Scientific and Technological Research Council of Turkey (TUBITAK) under research project No. 114E006.

\section{References}

[1] B. Maniscalco, P.M. Kaminski, J.M. Walls, Thin film thickness measurements using scanning white light interferometry, Thin Solid Films 550 (2014) 10-16, http://dx.doi.org/10.1016/j.tsf.2013.10.005.

[2] Y.-S. Ghim, S.-W. Kim, Thin-film thickness profile and its refractive index measurements by dispersive white-light interferometry, Opt. Express 14 (24) (2006) 11885-11891, http://dx.doi.org/10.1364/OE.14.011885.

[3] C.-Y. Han, Z.-Y. Lee, Y.-F. Chao, Determining thickness of films on a curved substrate by use of ellipsometric measurements, Appl. Opt. 48 (17) (2009) 3139-3143, http://dx.doi.org/10.1364/A0.48.003139.

[4] F.L. McCrackin, E. Passaglia, R.R. Stromberg, H.L. Steinberg, Treasure of the past vii: mMeasurement of the thickness and refractive index of very thin films and the optical properties of surfaces by ellipsometry, J. Res. Nat. Inst. Stand. Technol. 106 (3) (2001) 589-603, http://dx.doi.org/10.6028/jres.106.025.

[5] C. Kelb, M. Körner, O. Prucker, J. Rühe, E. Reithmeier, B. Roth, Pdmaa hydrogel coated u-bend humidity sensor suited for mass-production, Sensors 17 (3) (2017), http://dx.doi.org/10.3390/s17030517.

[6] L. Zhang, F. Gu, J. Lou, X. Yin, L. Tong, Fast detection of humidity with a subwavelengthdiameter fiber taper coated with gelatin film, Opt. Express 16 (17) (2008) 13349-13353, http://dx.doi.org/10.1364/OE.16.013349.

[7] C.R. Zamarreño, M. Hernáez, I. Del Villar, I.R. Matías, F.J. Arregui, Optical fiber ph sensor based on lossy-mode resonances by means of thin polymeric coatings, Sens. Actuators B: Chem. 155 (1) (2011) 290-297, http://dx.doi.org/ 10.1016/j.snb.2010.12.037.

[8] Y.M. Chen, Diffraction by a smooth transparent object, J. Math. Phys. 5 (6) (1964) 820-832, http://dx.doi.org/10.1063/1.1704183. 
[9] M. Amiri, M.T. Tavassoly, Fresnel diffraction from 1d and 2d phase steps in reflection and transmission modes, Opt. Commun. 272 (2) (2007) 349-361, http://dx.doi.org/10.1016/j.optcom.2006.11.048.

[10] A. Sabatyan, M.T. Tavassoly, Application of fresnel diffraction to nondestructive measurement of the refractive index of optical fibers, Opt. Eng. 46 (12) (2007) http://dx.doi.org/10.1117/1.2819745, 128001-1-128001-7.

[11] M.S. Dinleyici, C. Sümer, Characterization and estimation of refractive index profile of laser-written photopolymer optical waveguides, Opt. Commun. 284 (21) (2011) 5067-5071, http://dx.doi.org/10.1016/j.optcom.2011.07.038.
[12] L. Alwis, K. Bremer, T. Sun, K.T.V. Grattan, Analysis of the characteristics of pvacoated lpg-based sensors to coating thickness and changes in the external refractive index, IEEE Sens. J. 13 (3) (2013) 1117-1124, http://dx.doi.org/ 10.1109/JSEN.2012.2230534.

[13] R. Merlin, Radiationless electromagnetic interference: evanescent-field lenses and perfect focusing, Science 317 (5840) (2007) 927-929, http://dx.doi.org/ $10.1126 /$ science. 1143884 . 